\title{
BMJ Open Clinical, behavioural and social factors associated with racial disparities in COVID-19 patients from an integrated healthcare system in Georgia: a retrospective cohort study
}

\author{
Felipe Lobelo (1) , ${ }^{1,2}$ Alan Bienvenida, ${ }^{1}$ Serena Leung, ${ }^{1}$ Armand Mbanya, ${ }^{1}$ \\ Elizabeth Leslie, ${ }^{1}$ Kate Koplan, ${ }^{1}$ Sangmin Ryan Shin ${ }^{1}$
}

To cite: Lobelo F, Bienvenida A, Leung S, et al. Clinical, behavioural and social factors associated with racial disparities in COVID-19 patients from an integrated healthcare system in Georgia: a retrospective cohort study. BMJ Open 2021;11:e044052. doi:10.1136/ bmjopen-2020-044052

- Prepublication history for this paper is available online. To view these files, please visit the journal online (http://dx.doi. org/10.1136/bmjopen-2020044052).

Received 23 August 2020 Revised 19 February 2021 Accepted 06 April 2021
Check for updates

(C) Author(s) (or their employer(s)) 2021. Re-use permitted under CC BY-NC. No commercial re-use. See rights and permissions. Published by BMJ.

${ }^{1}$ The Southeast Permanente Medical Group, Kaiser Permanente Georgia, Atlanta, Georgia, USA

${ }^{2}$ Global Health Department, Rollins School of Public Health, Atlanta, Georgia, USA

Correspondence to Dr Felipe Lobelo; felipe.lobelo@kp.org

\section{ABSTRACT}

Objectives To identify sociodemographic, clinical and behavioural drivers of racial disparities and their association with clinical outcomes among Kaiser Permanente Georgia (KPGA) members with COVID-19. Design Retrospective cohort of patients with COVID-19 seen from 3 March to 29 October 2020. We described the distribution of underlying comorbidities, quality of care metrics, demographic and social determinants of health (SDOH) indicators across race groups. We also described clinical outcomes in hospitalised patients including length of stay, intensive care unit (ICU) admission, readmission and mortality. We performed multivariable analyses for hospitalisation risk among all patients with COVID-19 and stratifyied by race and sex.

Setting KPGA, an integrated healthcare system. Participants 5712 patients who all had laboratoryconfirmed COVID-19. Of them, $57.8 \%$ were female, $58.4 \%$ black, 29.5\% white, 8.5\% Hispanic and 3.6\% Asian. Results Black patients had the highest proportions of living in neighborhoods under the federal poverty line (12.4\%) and in more deprived locations (neighbourhood deprivation index=0.4). Overall, $14.4 \%(n=827)$ of this cohort was hospitalised. Asian patients had the highest rates of ICU admission (53.1\%) and mechanical ventilation (21.9\%). Among all patients, Hispanics (adjusted 1.60, $95 \% \mathrm{Cl}(1.08,2.37))$, blacks $(1.43(1.13,1.83))$, age in years $(1.03(1.02,1.04))$ and living in a zip code with high unemployment $(1.08(1.03,1.13))$ were associated with higher odds of hospitalisation. COVID-19 patients with chronic obstructive pulmonary disease $(2.59$ $(1.67,4.02))$, chronic heart failure $(1.79(1.31,2.45))$, immunocompromised $(1.77(1.16,2.70))$, with glycated haemoglobin $>8 \%(1.68(1.19,2.38))$, depression $(1.60(1.24,2.06))$, hypertension $(1.5(1.21,1.87))$ and physical inactivity $(1.25(1.03,1.51))$ had higher odds of hospitalisation.

Conclusions Black and Hispanic KPGA patients were at higher odds of hospitalisation, but not mortality, compared with other race groups. Beyond previously reported sociodemographics and comorbidities, factors such as quality of care, lifestyle behaviours and SDOH indicators should be considered when designing and

\section{Strengths and limitations of this study}

- In the USA and across the world, racial and ethnic minorities have shouldered a disproportionate burden of COVID-19 infection, but data on the various clinical and social drivers of these disparities are limited.

- As a limitation, the target population in this analysis included only Kaiser Permanente Georgia patients that have insurance and ready access to healthcare services.

- To our knowledge, this is the first COVID-19 retrospective cohort study to incorporate multiple individual and community-level social determinants of health indicators, pre-pandemic lifestyle behaviours and comorbidity management metrics as drivers of COVID-19 racial disparities.

implementing interventions to reduce COVID-19 racial disparities.

\section{INTRODUCTION}

As of 15 November 2020, the USA had over 10.5 million cases and 250000 deaths due to COVID-19. ${ }^{1}$ This accounts for $20 \%$ of the cases and deaths reported worldwide, despite the USA having about $4 \%$ of the global population. It has been widely reported that racial/ ethnic minorities, particularly those living in large and diverse urban centres, shoulder a disproportionate burden of the COVID-19 infection risk and associated adverse health outcomes. $^{2-6}$

Earlier descriptive studies from patients admitted during March/April 2020 in Georgia showed an over-representation of COVID-19 hospitalisations and death rates among black populations. ${ }^{7} 8$ Subsequent reports from two large healthcare systems in Louisiana and California, and from the 
Veterans Affairs health system ${ }^{9}$ also found racial disparities in COVID-19 outcomes and clinical risk factors for hospitalisation. These reports also theorised that chronic disease control, health behaviours, social and other factors may contribute to such disparities. ${ }^{3578}$ However, limited availability of quality of care history and social determinants of health $(\mathrm{SDOH})$ metrics in most medical health records has precluded a more comprehensive analysis of potential drivers of these racial disparities.

The US Census Bureau reports the racial/ethnic demographic distribution of Georgia as $57.8 \%$ white, $31.9 \%$ black, $4.1 \%$ Asian, $0.4 \%$ American Indian/Alaska Native, $2.7 \%$ two or more races and $3 \%$ some other race with $9.8 \%$ Hispanics (irrespective of race).$^{10}$ As of 20 November, the Georgia Department of Public Health (DPH) reported 399410 confirmed cases of COVID-19 with the following categorisation by race/ethnicity: $37 \%$ white, $27.5 \%$ black, 12.5\% Hispanic, $1.9 \%$ Asian, 2.6\% other race (American Indian/Alaska Native, Native Hawaiian/Pacific Islander) and $18.5 \%$ unknown or no data. ${ }^{11}$ This over-representation of black and Hispanic populations in terms of COVID-19 burden has also been observed in other US areas. ${ }^{46912-15}$ Kaiser Permanente Georgia (KPGA) is a regional integrated healthcare system serving over 300000 patients in 32 counties located in the Atlanta Metropolitan Area and Northeast Georgia. As of April 2020, KPGA membership is $43 \%$ black, $30 \%$ white, $5 \%$ Asian, $4 \%$ Hispanic and $18 \%$ unknown/other, which mirrors that of the Atlanta Metropolitan Area. ${ }^{16}$

This study had two objectives. First, to determine if racial disparities exist among KPGA patients with COVID-19, with respect to demographic and $\mathrm{SDOH}$, pre-pandemic comorbidities/underlying conditions, quality of care metrics and lifestyle behaviours as well as COVID-19related clinical outcomes (hospitalisation, intensive care unit (ICU) admission, length of stay, mechanical ventilation, readmission and mortality). Second, to explore the roles of these clinical, behavioural and $\mathrm{SDOH}$ factors as potential drivers of racial disparities for COVID-19 hospitalisation.

\section{METHODS}

We performed a retrospective review of KPGA patients seen with COVID-19-related symptoms between 3 March and 29 October 2020. Patients were screened according to the US Centers for Disease Control and Prevention (CDC) and Georgia DPH guidelines. ${ }^{17} 18$ Patients who met the criteria were tested for SARS-CoV-2 by PCR. For this analysis, we included any KPGA member with a documented diagnosis and/or laboratory-confirmed COVID-19 PCR test in their Electronic Health Record (EHR) which also integrates tests performed in facilities outside of our healthcare system. At the start of the epidemic, KPGA prioritised testing among symptomatic healthcare workers and symptomatic KPGA patients requiring hospital admission. In mid-April, testing was progressively expanded to high-risk symptomatic patients (based on clinical criteria ( $>65$ years, immunocompromise, chronic obstructive pulmonary disease (COPD), moderate-to-severe asthma, serious heart condition, body mass index $(\mathrm{BMI})>40$, diabetes, chronic kidney disease (CKD), liver disease, pregnancy) and symptomatic patients with public health implications (healthcare workers, first responders, jail and elder care employees, etc). Tests were offered in the following manner. After in-person or telemedicine evaluation, patients were tested, if recommended, via drive-thru and/or tents at one of four KPGA facilities located across metro Atlanta.

\section{Patient demographics}

We characterised patients with COVID-19 by age, sex, self-reported race/ethnicity, insurance type and area of residence. Race/ethnicity was categorised in our ER as African-American/Black (hereinafter referred to as 'black'), non-Hispanic white ('white'), Hispanic/Latino ('Hispanic'), Asian/Pacific Islander ('Asian'), 'unknown', 'declined to report' and 'Other', which included American Indian/Alaska Native. For purposes of this analysis, we excluded patients with COVID-19 seen during the study period in the 'Other' $(\mathrm{n}=13)$, 'unknown' $(\mathrm{n}=636)$ and 'declined to report' $(\mathrm{n}=95)$ categories, given the large heterogeneity of these groups and/or low sample size.

We obtained patient's location of residence and zip code from the EHR and categorised it into four different regions of metro Atlanta: Northeast, Northwest, Southeast and Southwest. Residence location was also linked to the Neighbourhood Deprivation Index (NDI), a composite SDOH measure including income, education, employment and housing quality. ${ }^{190}$ The higher the NDI value, the higher the level of deprivation in the neighbourhood. ${ }^{19}{ }^{20}$ We also used ESRI Business Analyst data, a comprehensive demographic and lifestyle database that provides data to help interpolate patient's socioeconomic status. ${ }^{21}$ Specifically, we linked patients' places of residency with ESRI's zip code level classifications of median household income, occupation (frontline, healthcare and other) and educational attainment. We used these data to cross-reference median household income with the government-defined poverty line. ${ }^{22}$

\section{Patient and public involvement}

Given the nature of this study, it was not appropriate or possible to involve patients or the public in the design, or conduct, or reporting or dissemination plans of our research.

\section{Comorbidities and quality of care (past 12 months)}

Existing comorbidities and clinical care of patients were obtained from the latest clinical visit dating back up to 12 months from the first COVID-19 encounter. Comorbidities were reported as classified by the International Statistical Classification of Diseases and Related Health Problems codes (ICD-10). ${ }^{23}$ The Deyo-Charlson Comorbidity Index (CCI) was used as a continuous measure of 
total comorbidity burden. ${ }^{24}$ The CGI is a weighted index developed to predict risk of death within 1 year of hospitalisation for patients with 17 specific common comorbidities. Each condition is assigned a weight from 1 to 6 , based on the estimated 1-year mortality HR and the weights summed to produce the CCI. A score of zero indicates no comorbidities, whereas the higher the score, the more comorbidity burden resulting in higher predicted mortality or resource utilisation.

We used pharmacy dispensing data to compile the frequency of outpatient medications used by patients. We used established clinical thresholds recommended by the National Committee for Quality Assurance as markers for adequate blood pressure $(<140 / 90 \mathrm{~mm} \mathrm{Hg})$ and (glycated haemoglobin (HbA1c) $<8 \%$ and $<9 \%$ ) blood glucose control. ${ }^{25}$

Exercise Vital Sign (EVS) data were collected and exercise minutes averaged from encounters in the 12 months prior to the first COVID related visit date. Patient's physical activity levels were classified as inactive, insufficiently active and sufficiently active for those self-reporting $\leq 10$, 11-149 and $\geq 150 \mathrm{~min}$ of exercise/week, respectively. The EVS has been previously validated ${ }^{26}$ and is considered a clinically relevant screening tool for physical activity behaviours in healthcare settings. ${ }^{27} 28$

\section{Clinical outcomes}

All patients with COVID-19 who were hospitalised at KPGA affiliated (2 core and 43 non-core) hospitals were characterised by hospital length of stay (LOS), intensive care unit (ICU) LOS, invasive mechanical ventilation initiation and duration, hospital discharge, 30-day and 60-day readmission, currently hospitalised and deceased. Hospital LOS consisted of the entire time spent in hospital from admission (including emergency department) to discharge (including death). Instances of admission and discharge on the same date were defined as LOS of 1 day. Mechanical ventilation data were compiled using ICD-10 code flagging instances and length of emergency endotracheal intubation during hospital stay. Readmissions were defined as instances of subsequent admission to a hospital within the KPGA health system due to COVID-19 complications or any other cause, 30 and 60 days after index discharge. We conducted manual record reviews to distinguish between encounters of readmission and patient transfers from a hospital to another non-KPGAaffiliated medical facility.

\section{Statistical analysis}

We report numbers (percentages) for binary and categorical variables and means (SD) for continuous variables. $\chi^{2}$ tests, analyses of variance (ANOVAs) and two sample t-tests were used to determine significant differences between groups. For two sample t-tests with statistically unequal variances, the Satterthwaite adjustment method was applied and reported.

Multivariable logistic regression was used to explore factors associated with having a COVID-19-related hospitalisation in seven different models: all COVID-19 patients, stratified by race/ethnicity (black, white, Hispanic, Asian) and by sex. All multivariable logistic regression models included age, sex and race/ethnicity as independent variables and hospitalisation as the dependent variable. All additional independent variables were assessed using a bivariate analysis, either $\chi^{2}$ or two sample t-test, and only the variables showing evidence of a statistically significant $(\alpha=0.05)$ relationship with the dependent variable were considered for entry into the models. A stepwise selection method was used for final independent variable selection with effect entry at significance levels of 0.05 and adjusted via the Student-Newman-Keuls post hoc test for differences in means. All data analysis was conducted using SAS V.9.4 software. All data relevant to the study are included in the manuscript.

\section{RESULTS}

\section{Demographic characteristics and SDOH}

Within the study period, we screened 52166 patients, tested $42421(81.3 \%)$ and 5721 (15.2\% of tested) patients were confirmed with COVID-19. The mean age of COVID-19-positive patients was 44.8 (15.7) years old (table 1). A higher proportion of black patients resided in neighbourhoods with a high proportion of households under the federal poverty level $(13.95 \%)$, with unfavourable NDI (0.37), and with the highest mean percentage of frontline $(35.6 \%)$ and healthcare workers $(7.4 \%)$ compared with other race groups (table 1). The highest overall percentage of the patients with COVID-19 resided in the Northeast Metro Atlanta area (36.5\%) (table 1). However, different areas of metro Atlanta showed varying prevalence of patients with COVID-19 when stratified by race/ethnicity. A higher proportion of black patients lived in the southern areas of metro Atlanta which visibly correlates with higher NDI neighbourhoods(figure 1).

\section{Comorbidities and quality of care}

Black patients had the highest rates of obesity (9\%), hypertension $(34.7 \%)$, asthma (11.3\%) and HIV (1.9\%), all $\mathrm{p}<0.0001$ (table 2). White patients presented with the highest rates of congestive heart failure (CHF; 7.2\%), coronary artery disease (CAD; 7.4\%), arrhythmia (5\%), chronic obstructive pulmonary disease (COPD; $4.6 \%$ ), depression $(15.5 \%)($ all $\mathrm{p}<0.0001)$ and overall CCI scores (2.1 (1.7)), $\mathrm{p}=0.0014)$ (table 2). Asian patients had the highest rate of diabetes (18\%; $\mathrm{p}=0.0022)$ (table 2). Compared with other race/ethnicity groups, black patients had the highest proportion of patients with uncontrolled blood pressure (BP) as defined by $\mathrm{BP}>140 / 90 \mathrm{~mm} \mathrm{Hg}$ $(30.1 \%)$ and the lowest self-reported mean $(\mathrm{SD})$ weekly exercise minutes (75.3 (113.4); $\mathrm{p}<0.0001)$ (table 2).

\section{Hospitalisation and other clinical outcomes}

Overall, 827 patients with COVID-19 were hospitalised with 896 hospital stays, a mean age of 57.3 (SD (15.8)) and an average length of stay of 7.9 (9.2) days 
Table 1 Sociodemographic characteristics of KPGA patients with COVID-19 seen from 3 March to 29 October 2020

KPGA members by race, $\mathrm{N}(\%)$

\begin{tabular}{|c|c|c|c|c|c|c|}
\hline & $\begin{array}{l}\text { All } \\
N=5721(100 \%)\end{array}$ & $\begin{array}{l}\text { Black } \\
n=3339(58.4 \%)\end{array}$ & $\begin{array}{l}\text { White } \\
n=1689(29.5 \%)\end{array}$ & $\begin{array}{l}\text { Hispanic } \\
n=487(8.5 \%)\end{array}$ & $\begin{array}{l}\text { Asian } \\
n=206(3.6 \%)\end{array}$ & $P$ value \\
\hline Age, mean (SD), years & $44.8(15.7)$ & $43.9(15.1)$ & $47.1(17.8)$ & $41.9(14)$ & $45.8(15.8)$ & $<0.0001$ \\
\hline \multicolumn{7}{|l|}{ Age range, years } \\
\hline $18-49$ & $3414(59.7)$ & $2106(63.1)$ & $865(51.21)$ & $328(67.4)$ & $115(55.8)$ & \multirow[t]{3}{*}{$<0.0001$} \\
\hline $50-64$ & $1686(29.5)$ & 931 (27.9) & 549 (32.5) & $140(28.8)$ & $66(32)$ & \\
\hline 65 and above & $621(10.9)$ & $302(9)$ & 275 (16.3) & 19 (3.9) & $25(12.1)$ & \\
\hline \multicolumn{6}{|l|}{ Gender } & \multirow[t]{3}{*}{$<0.0001$} \\
\hline Male & $2416(42.2)$ & $1270(38)$ & $820(48.5)$ & $226(46.4)$ & $100(48.5)$ & \\
\hline Female & 3304 (57.8) & $2068(62)$ & $869(51.5)$ & $261(53.6)$ & $106(51.5)$ & \\
\hline \multicolumn{7}{|l|}{ Insurance } \\
\hline Commercial & $4626(80.9)$ & $2675(80.1)$ & $1343(79.5)$ & $435(89.3)$ & $173(84)$ & \multirow[t]{5}{*}{$<0.0001$} \\
\hline Medicare & $567(9.9)$ & $291(8.7)$ & $245(14.5)$ & $14(2.9)$ & $17(8.3)$ & \\
\hline Medicaid & $6(0.1)$ & $4(0.1)$ & $0(0)$ & $2(0.4)$ & $0(0)$ & \\
\hline Self-pay & $326(5.7)$ & $237(7.1)$ & $59(3.5)$ & $17(3.5)$ & $13(6.3)$ & \\
\hline Other* & $196(3.4)$ & $132(4)$ & $42(2.5)$ & $19(3.9)$ & $3(1.5)$ & \\
\hline \multicolumn{7}{|l|}{ Median household income, $\mathrm{N}(\%) \dagger$} \\
\hline $25 k-50 k$ & 1079 (19.4) & $855(25.6)$ & $115(6.8)$ & $78(16)$ & $31(15)$ & \multirow[t]{4}{*}{$<0.0001$} \\
\hline $50 k-75 k$ & $2746(49.3)$ & $1814(54.3)$ & $639(37.8)$ & 225 (46.2) & $68(33)$ & \\
\hline $75 \mathrm{k}-100 \mathrm{k}$ & $1478(26.5)$ & $512(15.3)$ & $734(43.5)$ & $155(31.8)$ & $77(37.4)$ & \\
\hline $100 \mathrm{k}+$ & $272(4.9)$ & $58(1.7)$ & $170(10.1)$ & $19(3.9)$ & $25(12.1)$ & \\
\hline Households under poverty level, \%‡ & 12.36 & 13.95 & 9.85 & 11.96 & 10.37 & $<0.0001$ \\
\hline \multicolumn{7}{|l|}{ Residential region (\%)§ } \\
\hline Northeast & $2090(36.5)$ & $1085(32.5)$ & $626(37.1)$ & $274(56.3)$ & $105(51)$ & \multirow[t]{4}{*}{$<0.0001$} \\
\hline Northwest & 969 (16.9) & $341(10.2)$ & $492(29.1)$ & $102(20.9)$ & $34(16.5)$ & \\
\hline Southeast & $1116(19.5)$ & $854(25.6)$ & $192(11.4)$ & $35(7.2)$ & $35(17)$ & \\
\hline Southwest & $1179(20.6)$ & $822(24.6)$ & $280(16.6)$ & $53(10.9)$ & $24(11.7)$ & \\
\hline Neighbourhood Deprivation Index & 0.07 & 0.37 & -0.4 & 0.03 & -0.27 & $<0.0001$ \\
\hline \multicolumn{7}{|l|}{ Occupation, mean $\%{ }^{* *}$} \\
\hline Frontline workers & 33.6 & 35.6 & 30.2 & 35.4 & 31.2 & \multirow[t]{3}{*}{$<0.0001$} \\
\hline Healthcare workers & 7.2 & 7.4 & 7 & 6.5 & 6.6 & \\
\hline Other workers & 59.2 & 57 & 62.7 & 58.1 & 62.1 & \\
\hline \multicolumn{7}{|l|}{ Education, mean \%†† } \\
\hline Some high school & 6.6 & 7.2 & 5.8 & 7 & 5.8 & \multirow[t]{6}{*}{$<0.0001$} \\
\hline High school & 22.3 & 24 & 20.2 & 21.4 & 19.9 & \\
\hline Associates degree & 8.4 & 8.6 & 8.1 & 8.2 & 8.4 & \\
\hline Some college & 20.8 & 21.8 & 19.7 & 19.4 & 19.1 & \\
\hline Bachelors & 21.8 & 19.5 & 24.8 & 22.1 & 24.9 & \\
\hline Graduate & 12.6 & 11.5 & 14.2 & 12.3 & 14.4 & \\
\hline
\end{tabular}

*Other insurances include military Health Maintenance Organisation (HMO) or Preferred Provider Organisation (PPO).

†Based on ESRI Business Analyst dataset showing median household income by zip code and then linked to individual patients based on their recorded residence.

$\ddagger$ Poverty line was defined by the federal poverty level.

$\S$ The Atlanta metro area was divided up by county in four subregions.Northwest: Cobb, Cherokee, Paulding,Bartow, Pickens, Polk, Troup, Habersham.Northeast: Dekalb,

Gwinnett,Forsyth, Hall, Barrow, Jackson, Butts, Gilmer, Pike, Gordon, Jasper, Monroe.Southwest: Fulton, Douglas, Fayette,Coweta, Carroll, Meriwether, Heard, Dawson, Madison,

Lumpkin.Southeast: Clayton, Henry, Rockdale, Walton,Clarke, Spalding, Oconee, Muscogee, Brooks, Town.

IThe Neighbourhood Deprivation Index (NDI) is a composite measure of social and economic factors such as income, education, employment and housing quality that reflect neighbourhood deprivation. The higher the index value, the higher the level of deprivation in the neighbourhood.

${ }^{*}$ Based on ESRI Business Analyst data. Occupation Breakdown: Frontline workers included community/social services, protective services, food preparation/serving-related services, building/grounds cleaning/maintenance services, construction/extraction services, installation/maintenance/repair services, production services and transportation/material moving services. Healthcare workers included healthcare practitioners/technicians and healthcare support staff. Other workers included personal care/service workers, sales and sales-related workers, office/administrative support workers, farming/fishing/forestry workers, management/business/financial workers, computer/mathematical service workers, architecture/ engineering workers, life/physical/social science workers, community/social service workers, legal workers, education/training/library workers and arts/design/entertainment/sports/ media workers.

††Based on ESRI Business Analyst data. It is expressed in mean percentage and provides the counts of individual education attainment and occupation by category within each zip code (denominator).

KPGA, Kaiser Permanente Georgia. 


\section{Kaiser Permanente Georgia's COVID19 Cases By Race/Ethnicity}
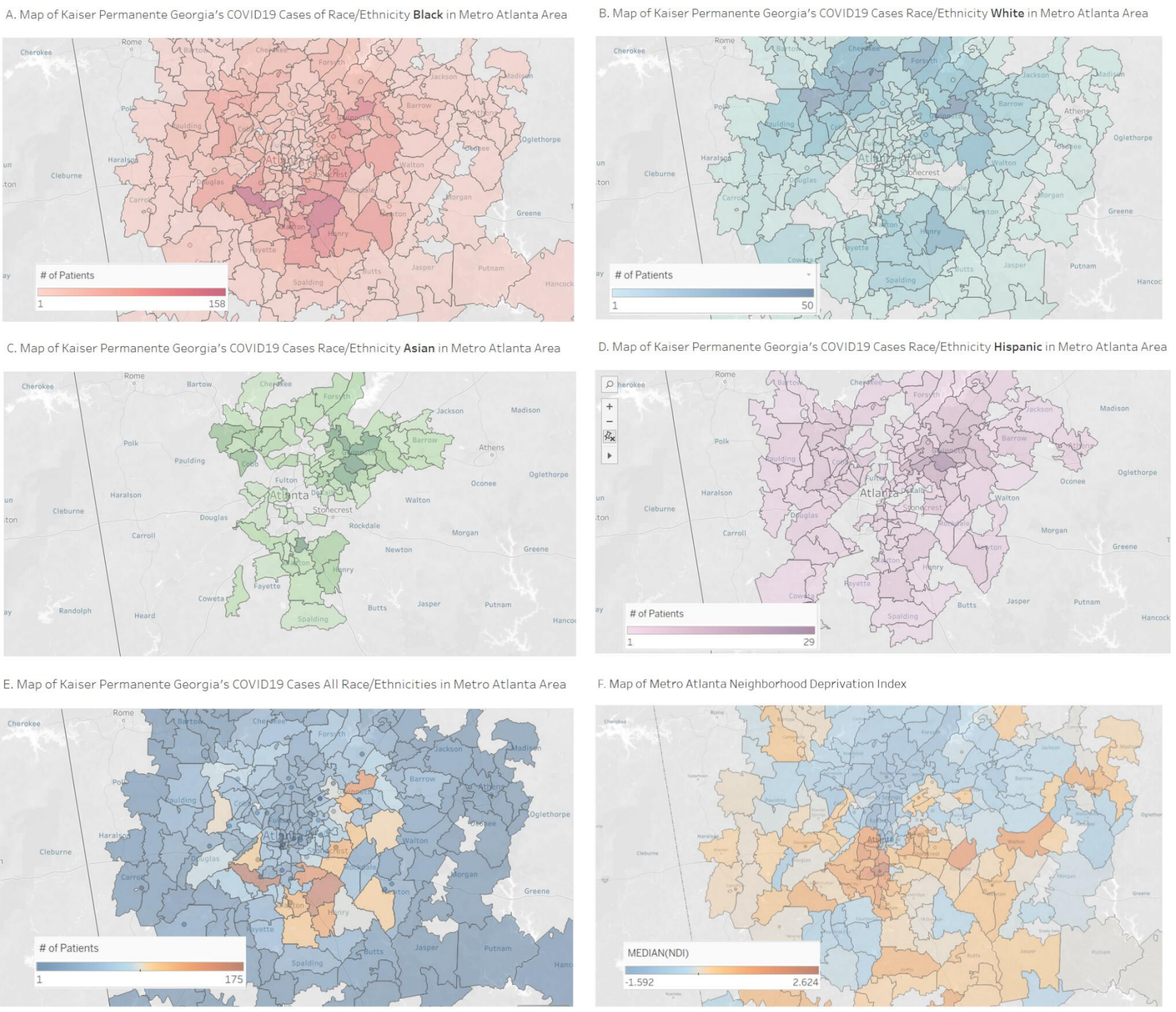

Figure 1 Map of Metro Atlanta Region's COVID-19 cases by race/ethnicity. (A) Map of COVID-19 cases: race black. (B) Map of COVID-19 cases: race white. (C) Map of COVID-19 cases: race Asian. (D) Map of COVID-19 cases: race Hispanic. (E) Map of COVID-19 cases: all races. (F) Map of Metro Atlanta Neighbourhood Deprivation Index (NDI).

(table 3). Of those hospitalised, $66 \%$ were admitted at our two core hospitals and $34 \%$ at non-core hospitals.

Compared with black and white patients, Asian patients had longer average hospital LOS (14.5 (17.1) vs $7.9(9.1), \mathrm{p}=0.0002$, and $7.2(7.9), \mathrm{p}<0.0001)$, ICU admission $(53.1 \%$ vs $27.7 \%, \mathrm{p}=0.0021$, and $27.6 \%$, $\mathrm{p}=0.0031)$ and invasive mechanical ventilation $(21.9 \%$ vs $9.2 \%, \mathrm{p}=0.0191$, and $5.8 \%, \mathrm{p}<0.0001)$, respectively (table 3). Asian patients also had a longer average hospital LOS than Hispanic patients (14.5 (17.1) vs 6.9 (7.3), $\mathrm{p}=0.0064)$. No significant differences in the rates of readmission or mortality were found between racial groups (table 3 ).

Male patients had longer average hospital LOS (8.6 (10.0) vs 7.3 (8.3), p=0.03) and higher rates of ICU admission (32.2\% vs 25.8\%; $\mathrm{p}=0.03)$ compared with female patients (table 3). Compared with patients aged 18-49 years, older patients aged 50-64 and 65+ had longer average hospital LOS (6.6 (8.2) vs 8.5 (9.9), $\mathrm{p}=0.0084$, and $8.5(9.2), \mathrm{p}=0.0092)$, higher rates of ICU admission (21.8\% vs $34.8 \%, \mathrm{p}<0.0001$, and $29.0 \%, \mathrm{p}=0.0265)$, invasive mechanical ventilation $(4.4 \%$ vs $10.9 \%, \mathrm{p}=0.0004$, and $9.9 \%, \mathrm{p}=0.0033)$ and death $(2.2 \%$ vs $8.1 \%, \mathrm{p}=0.001$, $21.1 \%, \mathrm{p}<0.0001$ ) (table 3 ). Of the 96 deceased patients in our cohort, $70 \%$ died during the COVID-19 index hospitalisation (table 3). Other patients died after discharge to hospice $(10.3 \%)$, assisted/skilled nursing facility or longterm assisted care $(9.3 \%)$, home $(7.3 \%)$ or other hospital $(3.1 \%)$. 
Table 2 Comorbidities, outpatient medication, quality of care and exercise metrics of KPGA patients with COVID-19, by race/ ethnicity

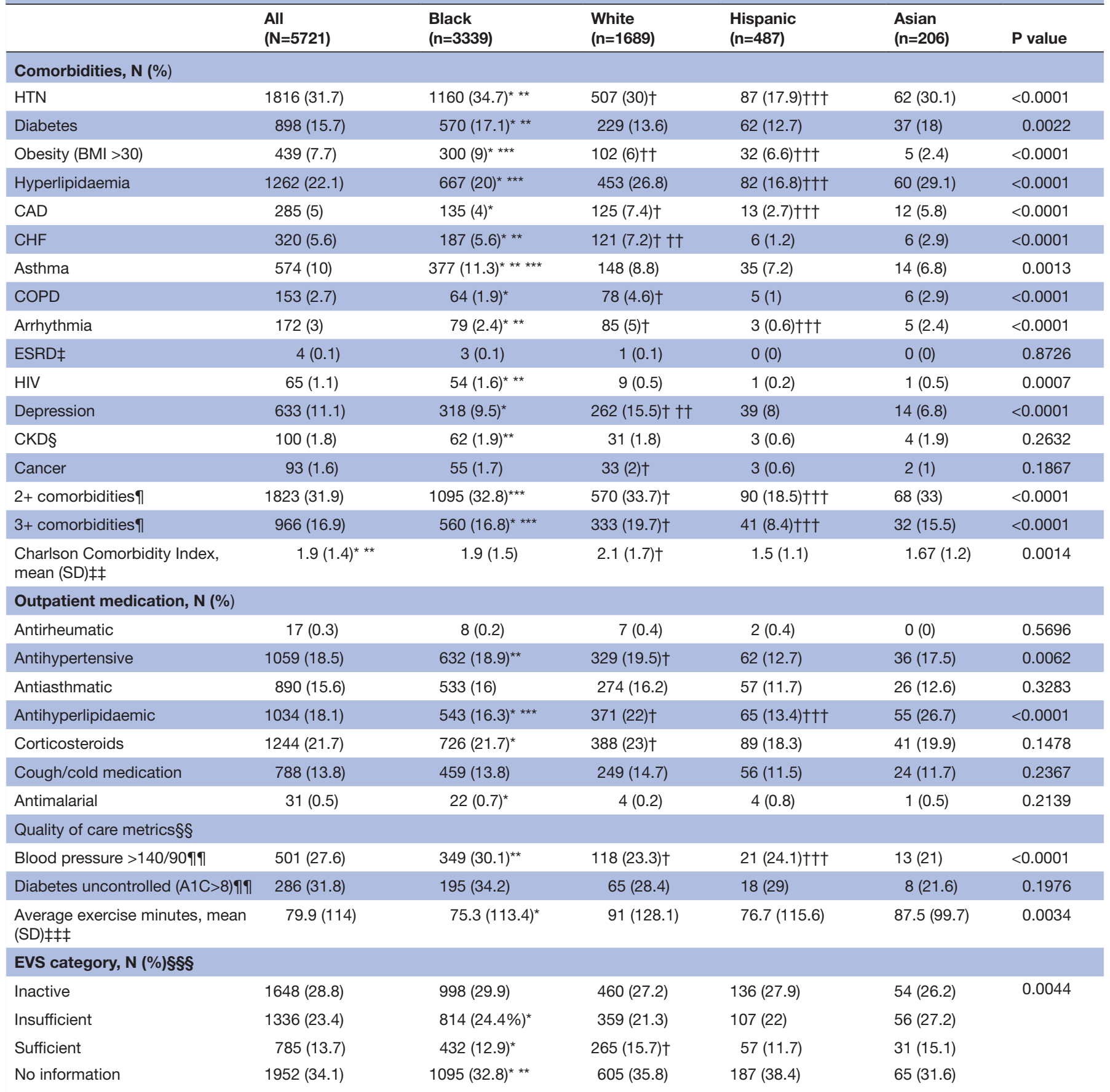

Significance levels: *Black vs white; ${ }^{* \star B}$ Black vs Hispanic; ${ }^{* \star *}$ Black vs Asian; †White vs Hispanic; ††White vs Asian; †††Hispanic vs Asian, significant difference at $\mathrm{p}<0.05$.

IESRD classified based on diagnosis reported by the 10th revision of the International Statistical Classification of Diseases and Related Health Problems code (ICD-

10) in patient's medical history.

§CKD classified based on diagnosis reported by the ICD-10 code in patient's medical history.

IC Comorbidities here are medical diagnoses included in medical history as ICD-10 codes. These include, but are not limited to, those presented in the table.

$\ddagger \ddagger C h a r l s o n$ Comorbidity Index predicts the 10-year mortality of a patient based on age and comorbidities. Scores are summed to provide a total predictive score. The lowest score of 0 corresponds to a $98 \%$ estimated 10-year survival rate (Charlson et al 1987).

$\S \S$ Assessed at the most recent clinical encounter within the last 12 months.

१ाBlood pressure control was evaluated only among patients with hypertension $(n=1816)$ and glucose control among patients with diabetes ( $n=898)$. $\ddagger \ddagger \ddagger$ Average exercise was collected from self-reported data.

$\S \S \S E V S$ is based on patient-reported weekly exercise minutes. We used three categories: inactive for patients who reported less than $10 \mathrm{~min} /$ week; insufficiently active for patients who reported 11-149 min/week; and sufficiently active for patients who reported 150 or more min/week.

$\mathrm{BMI}$, body mass index; CAD, coronary artery disease; CHF, congestive heart failure; CKD, chronic kidney disease; COPD, chronic obstructive pulmonary disease ESRD, end-stage renal disease; EVS, exercise as a vital sign; HbA1c, glycated haemoglobin; HTN, hypertension; KPGA, Kaiser Permanente Georgia. 


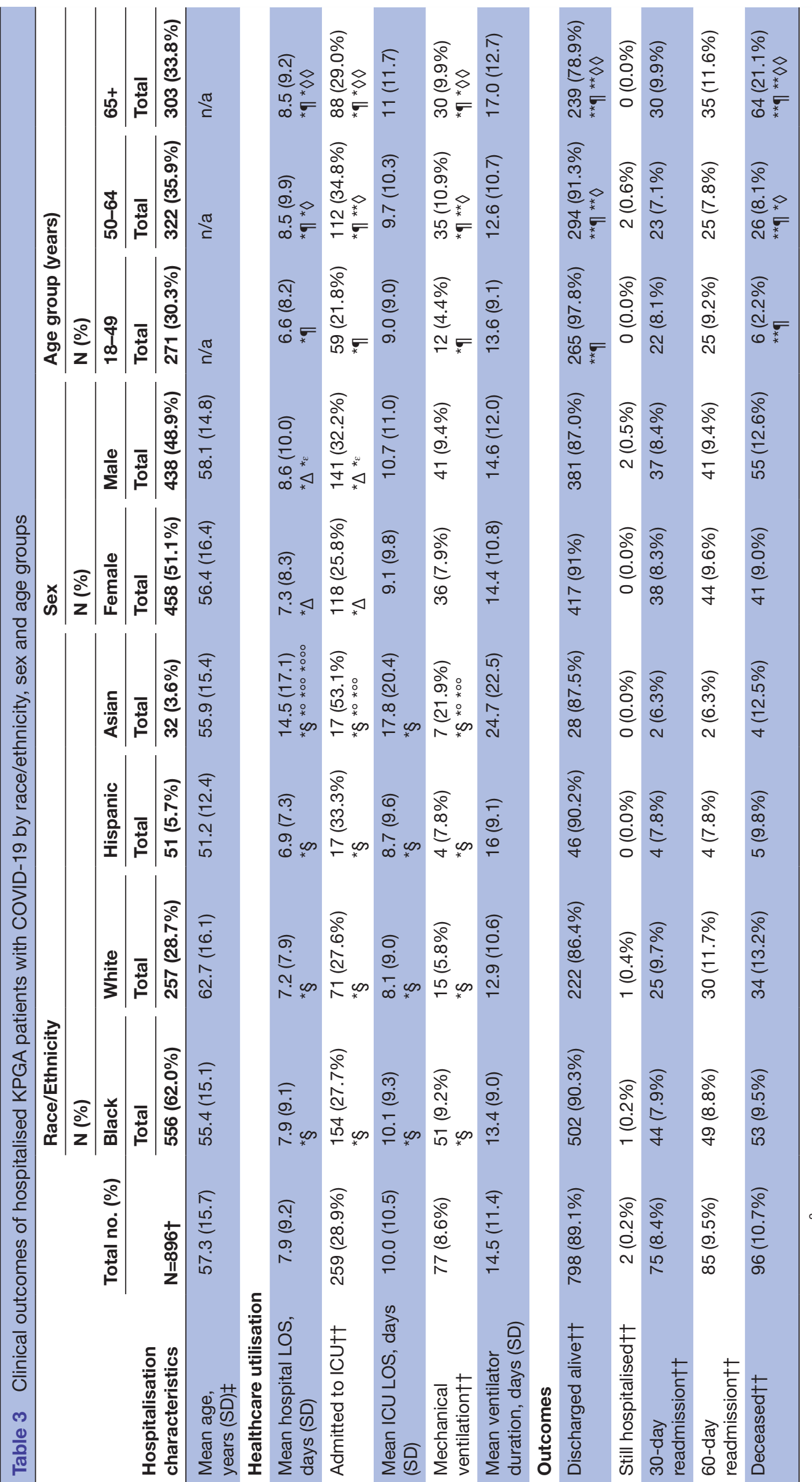

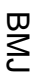

용

등

बె

次

응

N

के ᄒํ

齐

No

인

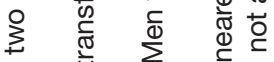

बं t

गु कू

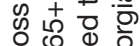

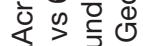
$\checkmark$ ष . <० 3 这递 这告 을

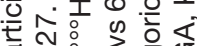
¿ II $=$ 9 \& की F 它这它

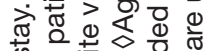

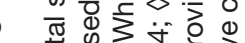

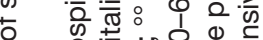

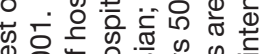
+0 운 ×

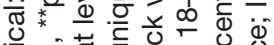

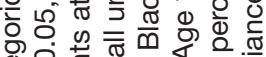

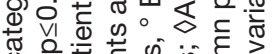
** व क क Ф 웅 \& 8 워요 का का का को

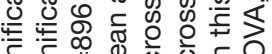

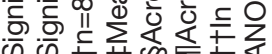




\section{Multivariable analysis and factors associated with hospitalisation \\ Overall model}

Sociodemographic factors including increasing age (adjusted OR (aOR) 1.02, 95\% CI (1.03, 1.04)), black race (aOR $1.43(1.13,1.83)$ ), Hispanic race (aOR $1.60(1.08,2.37))$, living in a zip code with high rates of unemployment (aOR $1.08(1.03,1.13)$ ) and having Medicare insurance (aOR $1.52 \quad(1.12,2.06)$ ) were associated with increased odds of hospitalisation for COVID-19 (table 4). Female sex was associated with lower odds of hospitalisation (aOR 0.74, 95\% CI (0.61, 0.90)) (table 4).

Comorbidities, quality of care and lifestyle factors associated with increased odds of hospitalisation included patients with COPD (aOR $2.59(1.67,4.02)$ ), CHF (aOR 1.79 (1.31, 2.45)), immunocompromised (aOR 1.77 $(1.16,2.70)$ ), with $\mathrm{HbA1c}>8 \%$ (aOR $1.68(1.19,2.38)$ ), depression (aOR $1.60(1.24,2.06)$ ), hypertension (aOR $1.5(1.21,1.87))$ and higher comorbidity scores (aOR 1.19 $(1.11,1.28))$ (table 4$)$. Additionally, self-reported physical inactivity was associated with higher odds of hospitalisation (aOR $1.25(1.03,1.51)$ ) (table 4).

\section{Race stratification}

Increasing age was associated with increased odds of hospitalisation across all race groups (table 4).

Among black patients, living in a zip code with high rates of unemployment (aOR $1.09(1.03,1.16)$ ) and having Medicare insurance (aOR $1.92(1.29,2.88)$ ) were associated with higher hospitalisation odds. Clinically, those with a history of COPD (aOR $2.53(1.24,5.16)$ ), CHF (aOR $2.19(1.47,3.27)$ ) and hypertension (aOR 1.74 $(1.30,2.32))$, as well as those with higher CCI (aOR 1.21 $(1.11,1.33)$ ), a recent (past 12 months) uncontrolled HbAlc $>8 \%($ aOR $1.74(1.13,2.66))$ or a cough/cold medication prescription (aOR $1.37(1.02,1.84)$ ), had higher odds of hospitalisation (table 4 ).

Among white patients, residence in northeast Atlanta (aOR $0.64(0.43,0.95))$ was protective for COVID-19 hospitalisation. White patients with a history of being immunocompromised (aOR $2.54(1.14,5.67)$ ), with COPD (aOR $2.49(1.38,4.49)$ ), depression (aOR 2.13 $(1.42,3.21))$, arrhythmia (aOR $1.89(1.05,3.42))$ and recent BP measurement $>140 / 90$ (aOR $2.17(1.31,3.57)$ ), as well as those with higher CCI (aOR $1.26(1.12,1.42)$ ) had increased odds of hospitalisation (table 4).

Among Hispanic patients, a recent uncontrolled HbA1c $(>8 \%)$ measurement was associated with higher odds of hospitalisation (aOR 5.95 (2.24, 15.78)) (table 4). Being a female was protective for hospitalisation with aOR 0.39 $(0.20,0.76)$ (table 4).

Among Asian patients, being a female (aOR 0.38 (0.15, $0.96)$ ) and residing in a high-income zip code (aOR 0.24 $(0.08,0.78))$ were protective against hospitalisation for COVID-19 (table 4).
Sex stratification

Increasing age was associated with increased odds of hospitalisation in all sex-stratified models (table 4).

Among male patients, residing in a high unemployment zip code was associated with increased odds of hospitalisation (aOR $1.11(1.04,1.19))$ (table 4). A recent uncontrolled $\mathrm{HbA1c}>9 \%$ (aOR $2.01(1.11,3.62)$ ), history of depression (aOR 1.73 (1.11, 2.69)), hypertension (aOR $1.58(1.15,2.17))$, recent antiasthmatic prescription (aOR $1.51(1.06,2.15))$ and a higher CCI (aOR 1.34 $(1.23,1.47))$ were clinical factors associated with higher hospitalisation odds (table 4).

Among female patients, sociodemographic factors associated with increased odds of hospitalisation included being black (aOR $1.46(1.06,2.02)$ ) and living in a high unemployment zip code (aOR 1.09 (1.02, 1.17)) (table 4). Clinical factors significantly associated with increased odds of hospitalisation were a history of COPD (aOR $4.34(2.42,7.77))$, CHF (aOR $2.62(1.67,4.12)$ ), immunocompromise (aOR $2.41(1.22,4.74)$ ), depression (aOR $1.52(1.11,2.09)$ ), uncontrolled $\mathrm{HbA} 1 \mathrm{c}>8 \%$ (aOR $1.76(1.07,2.90))$, hypertension (aOR $1.38(1.01,1.88)$ ), self-reported physical inactivity (aOR $1.45(1.12,1.89)$ ) and a higher CCI (aOR $1.12(1.01,1.24))$ (table 4).

\section{DISCUSSION}

This study reports an over-representation of black and Hispanic populations among the cohort of laboratoryconfirmed COVID-19 patients seen in an integrated care system serving the Southeast region of the USA. In comparison to the KPGA membership by race/ethnicity (43\% black, $30 \%$ white, $5 \%$ Asian, $4 \%$ Hispanic, $18 \%$ other/unknown), a higher proportion of black and Hispanic patients were diagnosed with COVID-19 (58.4\% and $8.5 \%$, respectively) and required hospitalisation $(62 \%$ and $5.7 \%$, respectively). White and Asian KPGA patients were not over-represented in terms of COVID-19 diagnosis $(29.5 \%$ and $3.6 \%$, respectively) or hospitalisation (28.7\% and 3.6\%, respectively). Although Asian patients showed significantly higher rates of disease severity (LOS, ICU admission, mechanical ventilation), we found no racial disparities in readmission or mortality rates.

Our findings are comparable to previous reports but with some important exceptions. Earlier studies have reported similar clinical outcomes between black and nonblack hospitalised COVID-19 patients in Georgia ${ }^{78}$ and some previous reports have also showed no differences in clinical outcomes between racial/ethnic groups. ${ }^{915}$ Asian patients have also been shown to present with a higher cardiorespiratory severity (aOR 1.48$)^{13}$ and be at 1.3 times increased risk of hospitalisation compared with white patients. ${ }^{15}$ National data from the CDC from August 2020 indicate that black and Hispanic patients were 4.6 and 4.7 times more likely than whites to be hospitalised for COVID-19. ${ }^{15}$ Other studies from academic or integrated healthcare systems have shown that after adjustment for age, sex, comorbidities and income, black patients had 


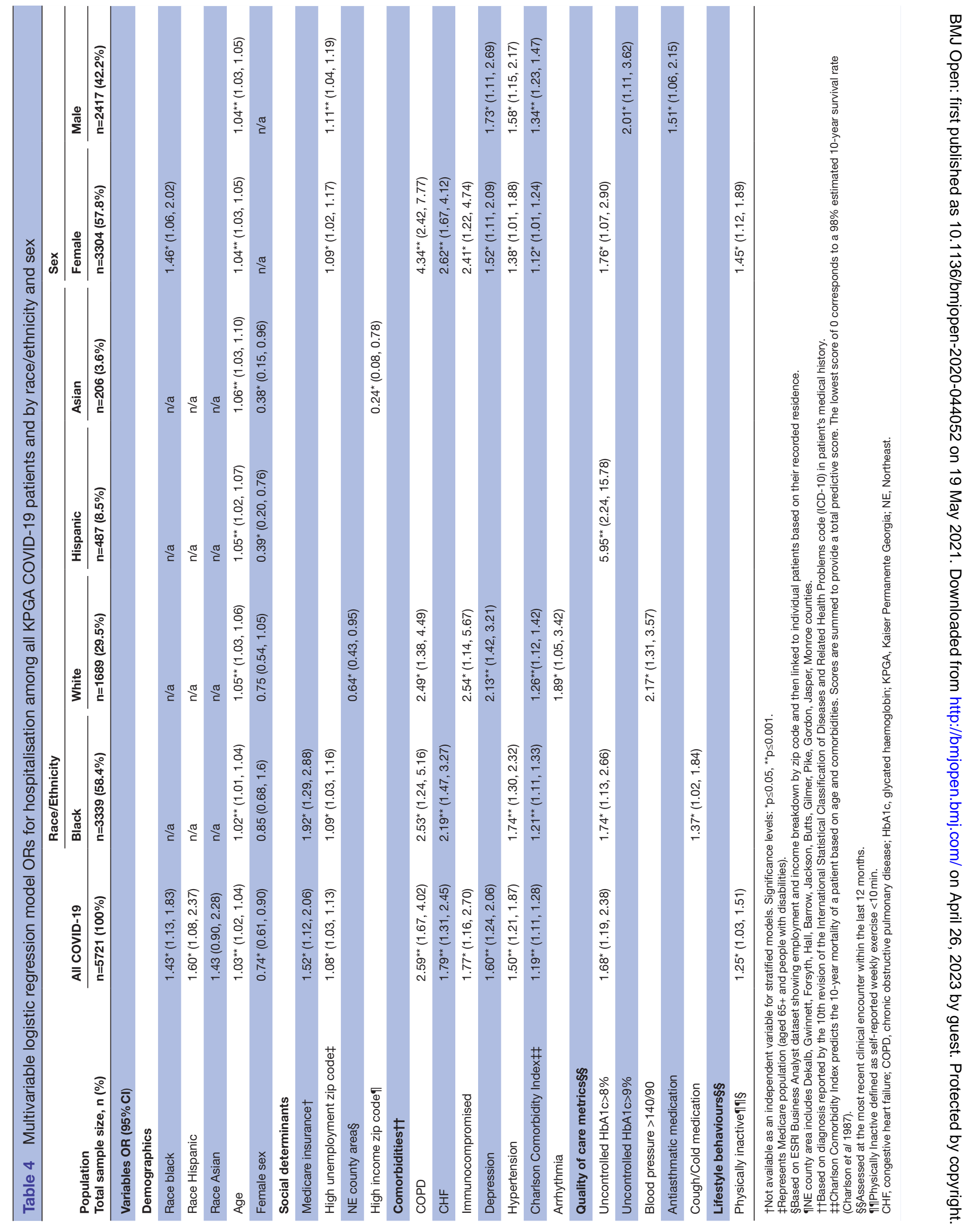


between 1.72 and 2.7 times and Hispanics 1.5 times the odds of hospitalisation compared with white patients. ${ }^{329} 30$

Compared with white patients, a higher percentage of black and Hispanic patients with COVID-19 were female, younger and more likely to reside in zip codes with a higher proportion of median household incomes below $\$ 75000$. Furthermore, black and Hispanic patients also resided at a higher proportion in neighbourhoods with the highest rate of households below the federal poverty level (14\% and $12 \%)$, with a higher NDI (0.37 and 0.03$)$, and the highest percentage of frontline workers $(35.6 \%$ and $35.4 \% \%$ ) compared with other racial groups. This and other SDOH factors have been associated with an increased risk of exposure to and infection with COVID-19 infection and underscore how systemic racism and inequities play a role in health disparities, a situation that has been magnified by the COVID-19 pandemic in the USA.

In addition to SDOH factors, comorbidities have been associated with more severe COVID-19 disease. The prevalence of comorbidities in the USA is inequitably distributed across race groups with minority populations shouldering a heavier burden of disease. Black patients had significantly higher prevalence of obesity, hypertension, asthma and HIV, all associated with increased disease severity in our analysis, as has been reported in previous studies. ${ }^{4}$ In our cohort, Asian patients had the highest diabetes prevalence. White patients were older, with higher CCI scores compared with black patients, and had a significantly higher prevalence of underlying conditions, such as hyperlipidaemia, CAD, CHF, COPD, arrhythmia and depression. Although there is a high prevalence of obesity, diabetes and other chronic diseases in the overall US population, particularly in the Southeast, ${ }^{31-33}$ our study and other reports suggest that different comorbidity phenotypes may influence COVID-19 disease severity across racial groups. ${ }^{13}$

Similar to previous studies, our multivariable analysis revealed that females were significantly (aOR 0.74 ) less likely to be hospitalised, while racial minorities (black, Hispanic) increasing age and chronic comorbidities were predominant factors associated with higher odds of hospitalisation. ${ }^{78}$ Medicare insurance type was a significant correlate of hospitalisation, a finding that was expected given the age of the population that has access to this insurance option. The median age for Medicare beneficiaries at KPGA was 71.8 vs 41.8 years for those with other types of insurance.

Interestingly, a recent uncontrolled blood glucose measurement $(\mathrm{HbA} 1 \mathrm{c}>8 \%)$ was an independent risk factor for hospitalisation among all patients (aOR 1.68), black (aOR 1.74) and particularly Hispanic patients (aOR 5.95). Furthermore, poor BP control $(>140 / 90 \mathrm{~mm} \mathrm{Hg})$ was a predictive factor for hospitalisation (aOR 2.17) among white patients. Overall, these findings suggest that presence of, and poorly controlled comorbidities, increase risk of hospitalisation for COVID-19 and that improving clinical management of underlying cardiometabolic diseases could help ameliorate hospitalisation rates. As the pandemic waves progresses over time, particular emphasis on implementing evidence-based strategies to reduce well-established racial disparities in diabetes and hypertension management ${ }^{2534}$ should be reinforced. Approaches that leverage novel avenues of care including telemedicine and patient-generated actionable data, as well as sustainable linkages with community resources, are recommended.$^{34}{ }^{35}$ Moreover, identifying the drivers of poorly controlled comorbidities in minority populations, particularly diabetes among Hispanic patients, may be particularly impactful given the high prevalence of both diabetes and COVID-19 risk of infection and hospitalisation among this group.

In addition to demographic factors and underlying comorbidity burden and management, our analyses also accounted for the potential role of additional SDOH, including indicators of education, economic stability, health insurance type, neighbourhood and physical environment as well as pre-pandemic lifestyle behaviours. Of these metrics, we found that residence in zip codes with a high proportion of unemployment was a consistent factor associated with increased hospitalisation risk for all patients (aOR 1.08 ) and specifically black patients (aOR 1.09), although with a smaller effect than other factors. In contrast, residence in zip codes with a high proportion of high-income individuals (aOR 0.24) and living in the Northeast area of metro Atlanta (0.64) were powerful protective factors against hospitalisation among white and Asian patients, respectively. Northeast Atlanta counties have consistently higher levels of median income, quality housing, green space, better safety and education and have a lower prevalence of obesity compared with the southern regions of KPGA's catchment area. ${ }^{36}{ }^{37}$ This is another reflection of how systemic factors perpetuate racial inequities and influence the risk of adverse health outcomes.

Furthermore, self-reported physical inactivity—engaging in less than $10 \mathrm{~min}$ of moderate to vigorous exercise/ week-increased by $25 \%$ the odds of hospitalisation among patients in our cohort. The effect estimate of physical inactivity was even more pronounced for female patients (aOR 1.45). Several biological mechanisms may explain this novel association. Physical inactivity is a consistent risk factor for a plethora of chronic diseases shown to also increase COVID-19 severity. ${ }^{38}$ Increased inactivity and sedentary time and related comorbidities are also associated with an increased low-grade chronic inflammatory state, ${ }^{39}$ which may contribute to the known increased systemic inflammatory effects of COVID-19. In addition to being a modulator of inflammation, regular moderate exercise is also an important immunomodulator, particularly of the virus-fighting cytotoxic immune response. ${ }^{40}$ This is reinforced by epidemiological studies showing a link between moderate-to-vigorous regular exercise and a lower risk of upper respiratory tract viral infections-including influenza and pneumonia-as well as improved vaccine responses. ${ }^{41}$ Although previous reports have shown that self-reported exercise is a predictor of clinical outcomes, ${ }^{28}$ it is noteworthy that physical inactivity remained a significant 
correlate of hospitalisation risk in our study population, after adjusting for traditional risk factors such as age, BMI, comorbidity burden and therapeutic management. This reinforces the clinical value of promoting fitness and an active lifestyle, preferably outdoors, to reduce the risk of infection and disease severity of a novel infectious agent such as SARS-COV-2. ${ }^{42}$

This study has some limitations. The study population included only KPGA patients that have access to insurance and, therefore, ready access to healthcare services. However, our analysis showed a diverse socioeconomic background of KPGA patients underscoring the role of various $\mathrm{SDOH}$ in relation to COVID-19 risk of infection and hospitalisation. We excluded 'Other' $(\mathrm{n}=13)$ and the 'declined to report' ( $\mathrm{n}=95)$ race/ethnicity categories from our analyses. Despite the robustness of KPGA's EHR data collection procedures and additional manual chart abstractions, we could not obtain data for an additional 636 patients with 'unknown' race/ethnicity and thus this group was also excluded from the analyses given their large heterogeneity and the difficulty to interpret findings or establish comparisons. In total, these groups constituted about $11 \%$ of patients with COVID-19 seen at KPGA during the study period, a smaller proportion than the unknown race/ethnicity category in the Georgia DPH $(18.5 \%)$. Finally, despite having some SDOH indicators in our member's EHR, we also included neighbourhood level data to extrapolate additional SDOH metrics. Well-established US studies examining COVID-19 racial disparities have included some, but not all, of the $\mathrm{SDOH}$ metrics we were able to include in our analyses. ${ }^{3} 5929$ Ongoing investigation of the drivers in COVID-19 racial disparities will benefit from including more individual level SDOH data. Despite these limitations, by integrating underlying chronic disease management history, outpatient information, hospitalisation, clinical outcomes and post-discharge follow-up data, this study provides one of the most comprehensive assessments of patients with COVID-19 in relation to racial/ethnic disparities.

To our knowledge, this investigation is the first COVID-19 retrospective cohort to include a multivariate analysis on multiple measures of SDOH and pre-pandemic comorbidity management. Our study suggests that, within our sample of KPGA patients with ready access to insurance and high quality of care in an integrated healthcare system, black and Hispanic patients were still being disproportionately affected by COVID-19 infection and risk of hospitalisation. However, we found no significant differences in clinical outcomes such as readmission or mortality across race/ethnicity groups. These outcomes are not very frequent, therefore these findings need to be corroborated on a larger sample size. Location of residence, a proxy for the overall community context of our patients, appears to be a factor strongly associated with increased hospitalisation risk among black patients. The SDOH have shown to contribute to a more unfavourable baseline health status and therefore can indirectly impact COVID-19 risk of hospitalisation and severity. ${ }^{6}$ In addition to age, sex, location of residence and presence of comorbidities, pre-pandemic self-reported exercise levels and underlying blood pressure and glucose control may also significantly impact hospitalisation risk in different race groups. Therefore, as interventions designed to reduce COVID-19 disparities and the systemic effects of racism $^{43}$ are implemented, we recommend that in addition to well-known clinical and quality of care variables, individual and community-level social factors and lifestyle health behaviours be considered by clinicians, healthcare systems ${ }^{44}$ and public health stakeholders.

\section{Twitter Felipe Lobelo @felipelobelo}

Acknowledgements Special thanks to all the providers and staff of The Southeast Permanente Medical Group and Kaiser Permanente Georgia.

Contributors Drs FL and SRS had full access to all the data in the study and take responsibility for the integrity of the data and the accuracy of the data analysis. Concept and design: SRS, FL, KEK. Acquisition, analysis or interpretation of data: FL, AXB, SL, ANM, EJL, SRS. Drafting of the manuscript: FL, SRS, AXB, SL, ANM, EJL. Critical revision of the manuscript for important intellectual content: FL, KEK, SRS. Statistical analysis: SL, EJL. Administrative, technical or material support: FL, KEK, SRS. Supervision: FL, KEK, SRS.

Funding The authors have not declared a specific grant for this research from any funding agency in the public, commercial or not-for-profit sectors.

Map disclaimer The depiction of boundaries on the map(s) in this article does not imply the expression of any opinion whatsoever on the part of BMJ (or any member of its group) concerning the legal status of any country, territory, jurisdiction or area or of its authorities. The map(s) are provided without any warranty of any kind, either express or implied.

Competing interests None declared.

Patient and public involvement Patients and/or the public were not involved in the design, or conduct, or reporting or dissemination plans of this research.

Patient consent for publication Not required.

Ethics approval The KPGA institutional review board approved this study with a waiver of informed consent.

Provenance and peer review Not commissioned; externally peer reviewed.

Data availability statement All data relevant to the study are included in the article or uploaded as supplemental information. All data relevant to the study are included in the article.

Open access This is an open access article distributed in accordance with the Creative Commons Attribution Non Commercial (CC BY-NC 4.0) license, which permits others to distribute, remix, adapt, build upon this work non-commercially, and license their derivative works on different terms, provided the original work is properly cited, appropriate credit is given, any changes made indicated, and the use is non-commercial. See: http://creativecommons.org/licenses/by-nc/4.0/.

ORCID iD

Felipe Lobelo http://orcid.org/0000-0003-4185-7193

\section{REFERENCES}

1 World Health Organization. Coronavirus disease (COVID-19) situation report November 17, 2020. Available: https://www.who.int/ publications/m/item/weekly-epidemiological-update-17-november2020

2 Price-Haywood EG, Burton J, Fort D, et al. Hospitalization and mortality among black patients and white patients with Covid-19. N Engl J Med 2020;382:2534-43.

3 Azar KMJ, Shen Z, Romanelli RJ, et al. Disparities in outcomes among COVID-19 patients in a large health care system in California. Health Aff 2020;39:1253-62.

4 Suleyman G, Fadel RA, Malette KM, et al. Clinical characteristics and morbidity associated with coronavirus disease 2019 in a series of patients in metropolitan Detroit. JAMA Netw Open 2020;3:e2012270. 
5 Millett GA, Jones AT, Benkeser D, et al. Assessing differential impacts of COVID-19 on black communities. Ann Epidemiol 2020;47:37-44.

6 Centers for Disease Control and Prevention. National center for immunization and respiratory diseases (NCIRD) DoVD. COVID-19 in racial and ethnic minority groups Atlanta, GA, 2020. Available: https://www.cdc.gov/coronavirus/2019-ncov/need-extraprecautions/racial-ethnic-minorities.html [Accessed 2 Jul 2020].

7 Gold JAW, Wong KK, Szablewski CM, et al. Characteristics and Clinical Outcomes of Adult Patients Hospitalized with COVID-19 - Georgia, March 2020. MMWR Morb Mortal Wkly Rep 2020;69:545-50.

8 Killerby ME, Link-Gelles R, Haight SC, et al. Characteristics Associated with Hospitalization Among Patients with COVID-19 - Metropolitan Atlanta, Georgia, March-April 2020. MMWR Morb Mortal Wkly Rep 2020;69:790-4.

9 Rentsch CT, Kidwai-Khan F, Tate JP. Covid-19 by race and ethnicity: a national cohort study of 6 million United States veterans. medRxiv.

10 United States Census Bureau. Georgia, 2020. Available: https:// data.census.gov/cedsci/profile?q=Georgia\&g=0400000US13\&tid= ACSDP1Y2018.DP05 [Accessed 17 Jun 2020].

11 Georgia Department of Public Health. Georgia department of public health daily status report, 2020. Available: https://dph.georgia.gov/ covid-19-daily-status-report [Accessed 20 Nov 2020].

12 Georgia Department of Public Health. Georgia department of public health daily status report, 2020. Available: https://dph.georgia.gov/ covid-19-daily-status-report [Accessed 12 May 2020]

13 Rodriguez F, Solomon N, de Lemos JA, et al. Racial and Ethnic Differences in Presentation and Outcomes for Patients Hospitalized with COVID-19: Findings from the American Heart Association's COVID-19 Cardiovascular Disease Registry. Circulation 2020;382.

14 Renelus BD, Khoury NC, Chandrasekaran K, et al. Racial disparities in COVID-19 hospitalization and in-hospital mortality at the height of the new York City pandemic. J Racial Ethn Health Disparities 2020:1-7.

15 Centers for Disease Control and Prevention. National center for immunization and respiratory diseases (NCIRD) DoVD. COVID-19 hospitalization and death by Race/Ethnicity, 2020.

16 Metro Atlanta Chamber. Profile of Metro Atlanta, 2020. Available: https://www.metroatlantachamber.com/resources/reports-andinformation/executive-profile [Accessed 25 Jun 2020].

17 National Center for Immunization and Respiratory Diseases (NCIRD) Division of Viral Diseases. Standard operating procedure (SOP) for triage of suspected COVID-19 patients in non-US healthcare settings: early identification and prevention of transmission during triage. Centers for Disease Control and Prevention, 2020. https://www.cdc. gov/coronavirus/2019-ncov/hcp/non-us-settings/sop-triage-preventtransmission.html

18 Georgia Department of Public Health. COVID-19: guidance for healthcare professionals 2020. Available: https://dph.georgia.gov/ covid-19-guidance-healthcare-professionals [Accessed 18 Jun 2020].

19 Andrews MR, Tamura K, Claudel SE, et al. Geospatial analysis of neighborhood deprivation index (NDI) for the United States by County. J Maps 2020;16:101-12.

20 Messer LC, Laraia BA, Kaufman JS, et al. The development of a standardized neighborhood deprivation index. J Urban Health 2006;83:1041-62.

21 ArcGIS. Esri Demographics - Tapestry Segmentation: ArcGIS, 2019. Available: https://doc.arcgis.com/en/esri-demographics/data/ tapestry-segmentation.htm [Accessed 20 Jun 2022].

22 Office of the Assistant Secretary for Planning and Evaluation. U.S. Federal Poverty Guidelines Used to Determine Financial Eligibility for Certain Federal Programs: U.S Department of Health \& Human Services, 2020. Available: https://aspe.hhs.gov/povertyguidelines 2020
23 World Health Organization. The ICD-10 classification of mental and behavioural disorders: diagnostic criteria for research, 1993.

24 Deyo RA, Cherkin DC, Ciol MA. Adapting a clinical comorbidity index for use with ICD-9-CM administrative databases. J Clin Epidemiol 1992;45:613-9.

25 National Committee for Quality Assurance (NCQA). HEDIS measures and technical resources, 2020. Available: https://www.ncqa.org/ hedis/measures/2020

26 Coleman KJ, Ngor E, Reynolds K, et al. Initial validation of an exercise "vital sign" in electronic medical records. Med Sci Sports Exerc 2012;44:2071-6.

27 Young DR, Coleman KJ, Ngor E, et al. Associations between physical activity and cardiometabolic risk factors assessed in a southern California health care system, 2010-2012. Prev Chronic Dis 2014:11:E219.

28 Lobelo F, Rohm Young D, Sallis R, et al. Routine assessment and promotion of physical activity in healthcare settings: a scientific statement from the American heart association. Circulation 2018;137:e495-522.

29 Gu T, Mack JA, Salvatore M, et al. Characteristics associated with racial/ethnic disparities in COVID-19 outcomes in an academic health care system. JAMA Netw Open 2020;3:e2025197.

30 Poulson M, Neufeld M, Geary A, et al. Intersectional disparities among Hispanic groups in COVID-19 outcomes. J Immigr Minor Health 2021;23:4-10.

31 Hales CM, Carroll MD, Fryar CD. Prevalence of obesity and severe obesity among adults: United States, 2017-2018. NCHS data brief. 360. Hyattsville, MD: National Center for Health Statistics, 2020.

32 Centers for Disease Control and Prevention. National diabetes statistics report A. GA: Centers for Disease Control and Prevention, U.S. Dept of Health and Human Services, 2020.

33 Clark A, Jit M, Warren-Gash C, et al. Global, regional, and national estimates of the population at increased risk of severe COVID-19 due to underlying health conditions in 2020: a modelling study. Lancet Glob Health 2020;8:e1003-17.

34 Ceriello A, Schnell O. COVID-19: considerations of diabetes and cardiovascular disease management. J Diabetes Sci Technol 2020;14:723-4

35 Shabto JM, Loerinc L, O'Keefe GA, et al. Characteristics and outcomes of COVID-19 positive patients with diabetes managed as outpatients. Diabetes Res Clin Pract 2020;164:108229.

36 Emory University. COVID-19 health equity interactive Dashboard, 2020. Available: https://covid19.emory.edu/ [Accessed 23 Jun 2020].

37 County Health Rankings \& Roadmaps. 2020 Georgia report, 2020. Available: https://www.countyhealthrankings.org/reports/statereports/2020-georgia-report [Accessed 23 Jun 2020].

38 Powell KE, King AC, Buchner DM, et al. The scientific foundation for the physical activity guidelines for Americans, 2nd edition. $J$ Phys Act Health 2018:1-11.

39 Henson J, Yates T, Edwardson CL, et al. Sedentary time and markers of chronic low-grade inflammation in a high risk population. PLoS One 2013;8:e78350.

40 Nieman DC, Wentz LM. The compelling link between physical activity and the body's defense system. J Sport Health Sci 2019;8:201-17.

41 Song $Y$, Ren F, Sun D, et al. Benefits of exercise on influenza or pneumonia in older adults: a systematic review. Int $J$ Environ Res Public Health 2020;17:2655.

42 Sallis J, Pratt M. A call to action: physical activity and COVID-19 exercise is medicine, 2020. Available: https://www. exerciseismedicine.org/support_page.php/stories/?b=896 [Accessed 23 Jun 2020].

43 Bryan A D-GJ, Davis NJ, Chokshi DA. Moving from the five whys to five Hows: addressing racial inequities in COVID-19 infection and death. Health Aff 2020.

44 Parodi SCB, Young S, Bellows J. Kaiser Permanente's system capabilities to suppress Covid-19. NEJM Catal. 